\title{
Cultural adaptation and validation of patient decision aids: a scoping review
}

This article was published in the following Dove Press journal:

Patient Preference and Adherence

\author{
Vanessa Chenel ${ }^{1,2}$ \\ W Ben Mortenson ${ }^{3-5}$ \\ Manon Guay 6,7 \\ Jeffrey William Jutai ${ }^{8,9}$ \\ Claudine Auger ${ }^{1,2}$
}

'School of Rehabilitation, Faculty of Medicine, Université de Montréal,

${ }^{2}$ Centre for Interdisciplinary Research in Rehabilitation of Greater Montreal (CRIR) - Institut universitaire sur la réadaptation en déficience physique de Montréal, Centre intégré universitaire de santé et de services sociaux du Centre-Sudde-l'Île-de-Montréal, Montreal, QC, ${ }^{3}$ Department of Occupational Science and Occupational Therapy, Faculty of Medicine, University of British Columbia, ${ }^{4}$ GF Strong Rehabilitation Centre, ${ }^{5}$ International Collaboration on Repair Discoveries, University of British Columbia, Vancouver Coastal Health Research Institute, Vancouver, BC, ${ }^{6}$ School of Rehabilitation, Faculty of Medicine and Health Sciences, Université de Sherbrooke, ${ }^{7}$ Research Centre on Aging, Centre intégré universitaire de santé et de services sociaux de l'Estrie Centre hospitalier universitaire de Sherbrooke, Sherbrooke, QC, ${ }^{8}$ Interdisciplinary School of Health Sciences, University of Ottawa, ${ }^{9}$ Bruyère Research Institute, Ottawa, ON, Canada

Correspondence: Vanessa Chenel School of Rehabilitation, Faculty of Medicine, Université de Montréal, 7077 Parc Avenue, Montreal, QC H3N IX7, Canada

$\mathrm{Tel}+\mathrm{I} 5$ I4 3436 III (ext I7266)

Fax + I 5143432105

Email vanessa.chenel@umontreal.ca

\begin{abstract}
In order to promote self-determination, patients have to be actively involved with their care providers in health-care decision making, especially when such decisions involve personal preferences. Decision aids (DAs) are tools that can contribute to patient-centered decision-making processes. To benefit from previous fieldwork and avoid duplicating developmental efforts and producing many similar DAs, the adaptation of existing DAs to new cultural contexts is a resource-saving option. However, there are no guidelines on how to culturally adapt and validate DAs. This study aimed to identify and document existing procedures for the cultural adaptation and validation of patient DAs. A scoping review examined studies conducting cultural adaptation and/or validation of patient DAs. The following databases were searched in February 2016: CINAHL, EMBASE, Medline (Ovid), PASCAL, PsychINFO, and PubMed. From the 13 studies selected, 11 main procedures were identified: appraisal of the original DA, assessment of the new cultural context, translation, linguistic adaptation, cultural adaptation, usability testing, exploration of DA acceptability, test-retest reliability, content validity, construct validity, and criterion validity. A conceptual synthesis of these studies suggests there are four phases in the adaptation/validation process of DAs aimed at: 1) exploring the original DA and the new cultural context, 2) adapting the original DA to the new cultural context, 3) lab testing the preliminary version of the adapted DA, and 4) field testing the adapted DA in a real use context. By facilitating the adaptation and broader implementation of DAs, patients may ultimately be empowered in decision-making processes.
\end{abstract}

Keywords: decision making, decision support techniques, translation, cultural adaptation, validation studies

\section{Introduction}

Patient decision aids (DAs) are evidence-based tools designed to help patients make specific and deliberate choices among different health care options. ${ }^{1}$ DAs support decision-making procedures in contexts where multiple factors, and uncertainties, may influence the outcome. ${ }^{2}$ The use of patient DAs has had an important impact on medical decision-making processes. ${ }^{3}$ For example, by encouraging patient autonomy and self-determination, ${ }^{4}$ DAs contribute to the decision-making process by actively involving patients in preference-sensitive health-related choices. Operationally, DAs are interventions designed to define and frame the decision that needs to be made by providing evidence-based information about a health condition, the options, associated benefits, harms, probabilities, and scientific uncertainties. ${ }^{1}$ DAs help patients to recognize the value-added nature of the decision and clarify how they interpret the benefits, harms, and scientific uncertainties. By providing appropriate or even personalized information, DAs foster patient involvement. ${ }^{5}$ 
Many DAs have recently been developed to support client-centered practices. ${ }^{6,7}$ Adapting these DAs for other cultural settings could be a solution for other countries to such issues as scarce resources and the geographical dispersion of populations. However, DAs are developed for specific health-related or medical contexts and can be created in a generic form or target a precise population. ${ }^{8}$ Whenever DAs are developed with targeted content (eg, language, value-based framing of information, evidentiary information relevant to a cultural group), their use is optimal in the original context and amendments are necessary to make the material suitable in another cultural context. Depending on their complexity, the time and costs required for developing DAs can be considerable. ${ }^{9,10}$ Consequently, as is already done with health questionnaires, adapting existing DAs rather than developing new ones could be a way to benefit from previous fieldwork and avoid duplicating developmental efforts and producing many similar DAs. ${ }^{11}$

Cultural adaptation is defined as a process that looks at both language (translation) and cultural issues in the process of preparing a tool for use in another setting. ${ }^{12}$ Various guidelines were proposed in the 1990s regarding how to culturally adapt and validate health questionnaires. ${ }^{11-15}$ DAs are reflective and orientation tools involving evidence/information of relevance to the patient that can be used to reach a decision, rather than evaluation or measurement tools to be used by others. Thus, traditional guidelines for adapting healthrelated questionnaires are not optimally suited to the content and purpose of DAs. However, little information is available about how to culturally adapt and validate existing DAs.

There are some existing resources for the cultural targeting and tailoring of health-related education programs and interventions such as DAs. ${ }^{8,16}$ Kreuter et al defined cultural targeting as:

[...] the use of a single intervention approach for a defined population subgroup that takes into account characteristics shared by the subgroup's members. ${ }^{16}$

They also defined tailoring as:

[...] any combination of information intended to reach one specific person, based on characteristics that are unique to that person, related to the outcome of interest, and have been derived from an individual assessment. ${ }^{16}$

While these definitions are central to differentiating how a DA can target a specific population (targeting) and provide personalized information/recommendations (tailoring), they are limited to a description of principles related to cultural appropriateness when planning an intervention program.
Aligned with these definitions, Alden et al suggested a twostage targeting/tailoring DA design framework. ${ }^{8}$ Although these authors present a broad set of cultural elements to consider when developing a DA, ranging from colors and fonts to language, values and even evidence on the topic addressed, they offer little guidance on how to proceed when adapting an existing DA for another cultural setting.

Finally, clear instructions on how to develop patient DAs and assess the quality of existing DAs are available. ${ }^{6,10,17}$ The International Patient Decision Aid Standards (IPDAS) checklist is an internationally approved set of criteria to guide the development of new DAs and assess the quality of existing DAs. ${ }^{18}$ Composed of 12 quality domains including 74 dimensions, the objective of this tool is to improve the quality and effectiveness of DAs. Other models and maps describe the phases common to the development of patient DAs, including phases for scoping and content specification, designing and developing a prototype, and field testing the DA in different settings. ${ }^{6,10}$ However, although these guidelines include some considerations regarding cultural aspects pertaining to DAs under development, they are of little help in identifying concrete procedures for adapting DAs in different cultural contexts.

Given the need to culturally adapt DAs and then validate the adapted versions, and the lack of guidelines and methods, a study was undertaken to identify existing methods and key procedures for the cultural adaptation and validation of patient DAs.

\section{Methods}

To address the study objectives, a scoping review was conducted, which included a conceptual synthesis, according to the procedures outlined by Arksey and O'Malley and Levac et al. ${ }^{19,20}$ Distinct from the other types of literature reviews (eg, meta-analyses, systematic reviews), scoping reviews are intended to "map" the relevant literature in a field of interest when 1) there are many different study designs that might be applicable; 2) the quality of the studies is not central; and 3) the search does not focus on a very precise research question. ${ }^{19}$

\section{Search strategy}

The search strategy was developed with the assistance of a health care librarian, and articles were obtained from the following databases: CINAHL, EMBASE, Medline (Ovid), PASCAL, PsychINFO, PubMed. Keywords used for the search included: 1) "decision aid" or "decision support system" or "decision-making tool", and 2) validation or adaptation, and 3) patient or client or consumer or stakeholder. The search was updated until October 2017 and was limited to primary source articles written in English 
or French. Reference lists of selected articles were also reviewed.

\section{Study selection}

Studies were selected by the first author. Articles were included if they 1) were a primary source study, 2) described the adaptation or validation process of a DA, 3) described a DA intended to be used by patients, and 4) were peerreviewed. Articles were excluded if they 1) described a clinical DA (intended to be used by a health professional only), 2) validated the effectiveness or usefulness of an intervention based on a DA (and not the adaptation or validation process of the DA itself), or 3) were conference abstracts or poster submissions. The final inclusion and exclusion of full-text articles was confirmed by the last author.

\section{Synthesis of the evidence}

Information regarding selected studies was extracted by the first author and summarized in a table that included author(s), publication year, location of the study, research objectives, type of DA (based on an existing classification), ${ }^{21}$ DA format and purpose, and methods. The accuracy of the data extracted was confirmed by the last author. Content analysis was used to identify the main procedures and core phases structuring the cultural adaptation and validation process. ${ }^{22}$ While some procedures were identified based on existing frameworks for cross-cultural adaptation of health questionnaires ${ }^{11,12}$ and on existing guidelines for the quality of health measurement instruments, ${ }^{14}$ other procedures, including the core phases of the adaptation and validation process, were identified inductively. The first author initiated the synthesis of the methods, presented the results to the other authors, facilitated the discussions, and adjusted the proposed approach in the course of the various deliberations. The discussions continued until consensus was reached on the content to analyze.

\section{Findings}

\section{Search results}

The search is shown in Figure 1 in accordance with the Preferred Reporting Items for Systematic reviews and Meta-Analyses guidelines. ${ }^{23}$ The search process yielded 1,187 articles, which were imported into EndNote (Thomson Reuters, X7.1). From the 1,187 articles, 748 remained after removing the duplicates and the incomplete records. The 748 articles were screened to target only the ones related to the adaptation and the validation of patient DAs.

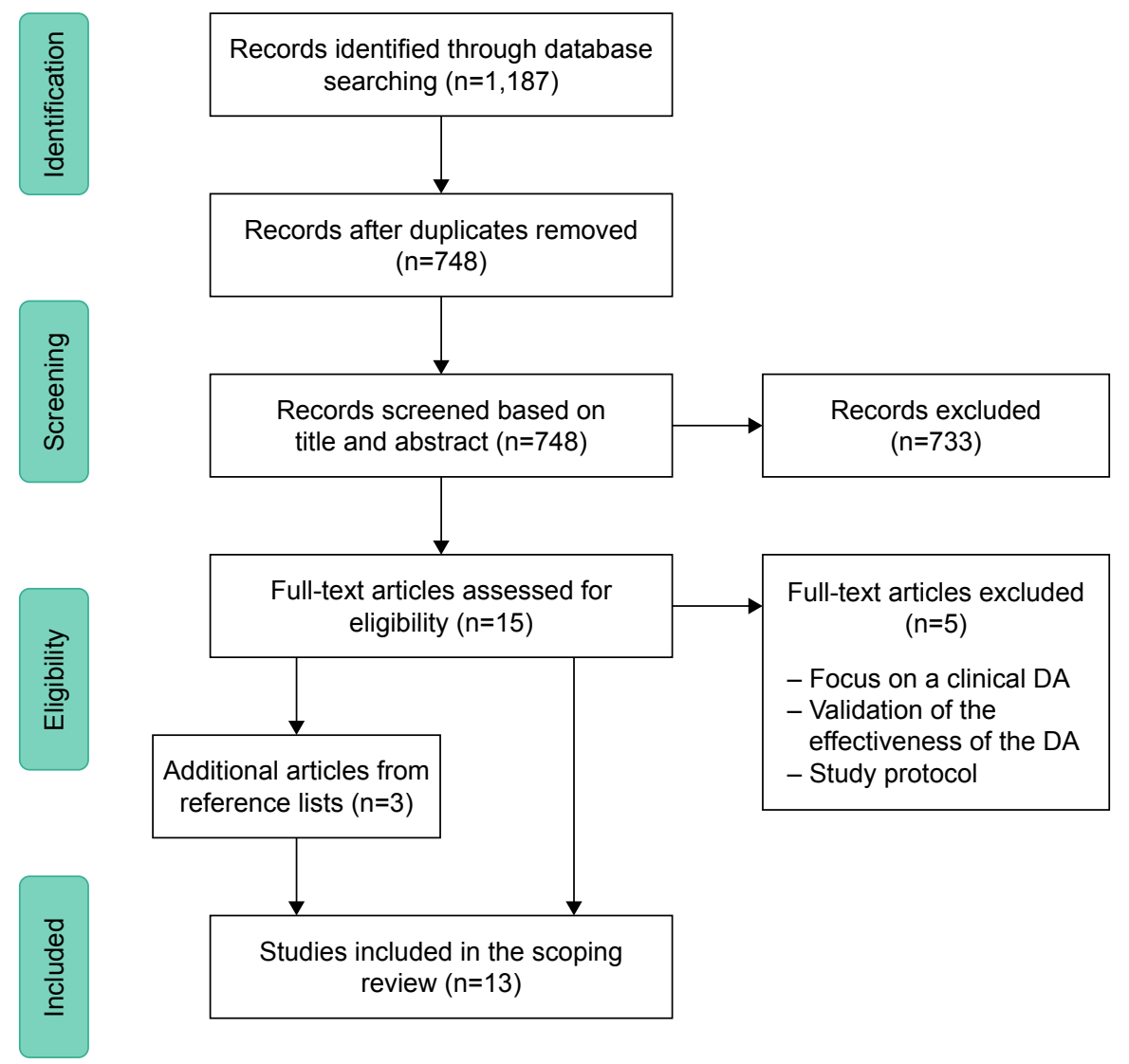

Figure I Flowchart of the search and the selection process. 
Most ( $n=733$ ) were excluded based on the title and abstract for not responding to the inclusion criteria (eg, related to a clinical DA, presenting an educational tool that was not a DA). Conference abstracts were also excluded. Of the 15 articles read in full to determine eligibility, 10 were included. Three articles were added via a review of reference lists of retained articles.

Selected studies are summarized in Table 1. Eleven articles were published over an 11-year period (2005-2016). All of the studies were conducted in four countries, namely the US ( $n=8)$, France $(n=2)$, Canada $(n=2)$, and the Netherlands $(n=1)$. Nine studies described the validation of a new DA, three the cultural adaptation of an existing DA, and one the entire cross-cultural development and validation of a DA. Various DA formats were found, including computer-based $(\mathrm{n}=7)$, material used in an interpersonal counseling program $(n=2)$, written material $(n=2)$, video clip $(n=1)$, and oral presentation of information without counseling $(n=1)$. All of the studies aimed mainly to provide understandable information and/or elicit patient preferences to enable them to make an informed choice regarding a specific medical context.

\section{Adaptation and validation methods}

From the 13 studies selected, 11 main procedures for the cultural adaptation and validation of patient DAs were identified: appraisal of the original DA; assessment of the new cultural context; translation; linguistic adaptation; cultural adaptation; usability testing; exploration of DA acceptability; test-retest reliability; content validity; construct validity; and criterion validity (see Table 1). The procedures identified are discussed later based on how they were applied in the adaptation and validation process of patient DAs.

\section{Appraisal of the original DA}

To prepare for the adaptation process, some researchers did an in-depth evaluation of the DA content and structure. ${ }^{24,25}$ For instance, Berry et al documented the DA structure and content to illustrate what needed to be adapted. ${ }^{25}$ Ko et al went a step further and also reviewed the associated published data to identify the structure and format of the original DA, the rationale, concepts, and theoretical underpinnings. ${ }^{24}$

\section{Assessment of the new cultural context}

The assessment of the new cultural context for the DA was reported as an important procedure in two studies. ${ }^{24,26} \mathrm{Ko}$ et al suggested three steps in this regard: review the relevant literature about the cultural context, involve stakeholders, and solicit input from the target population to guide the adaptation of the existing DA. ${ }^{24}$ Jull et al applied the same principles

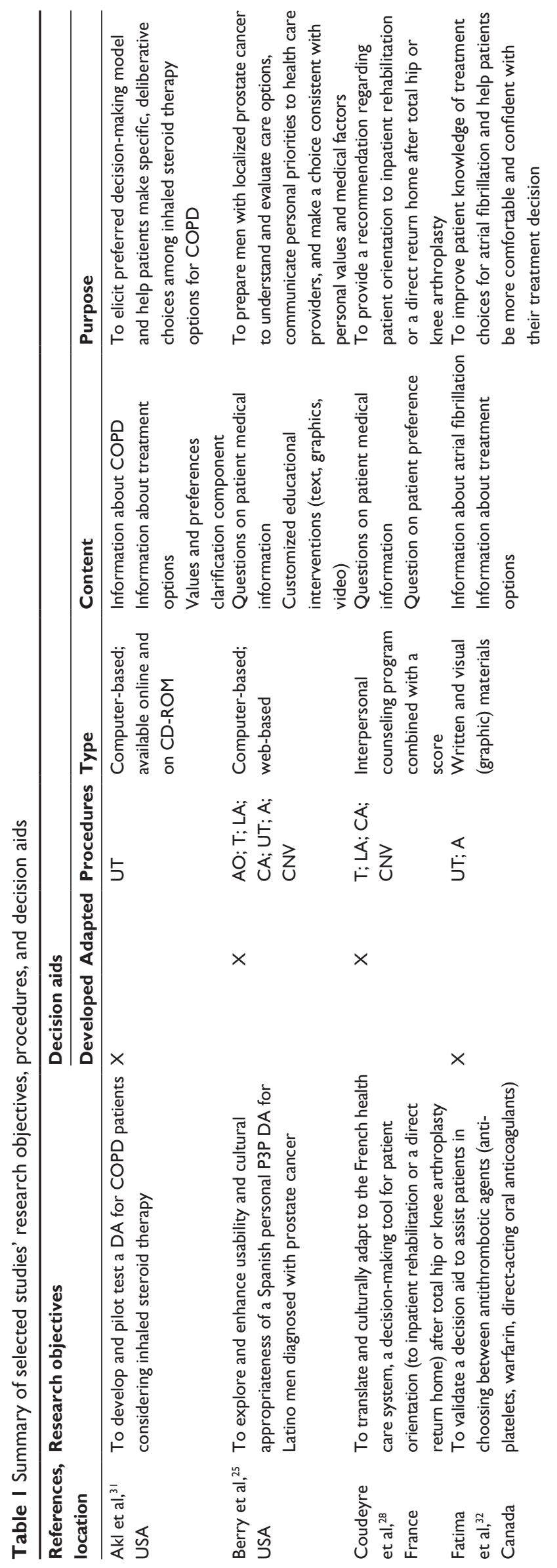



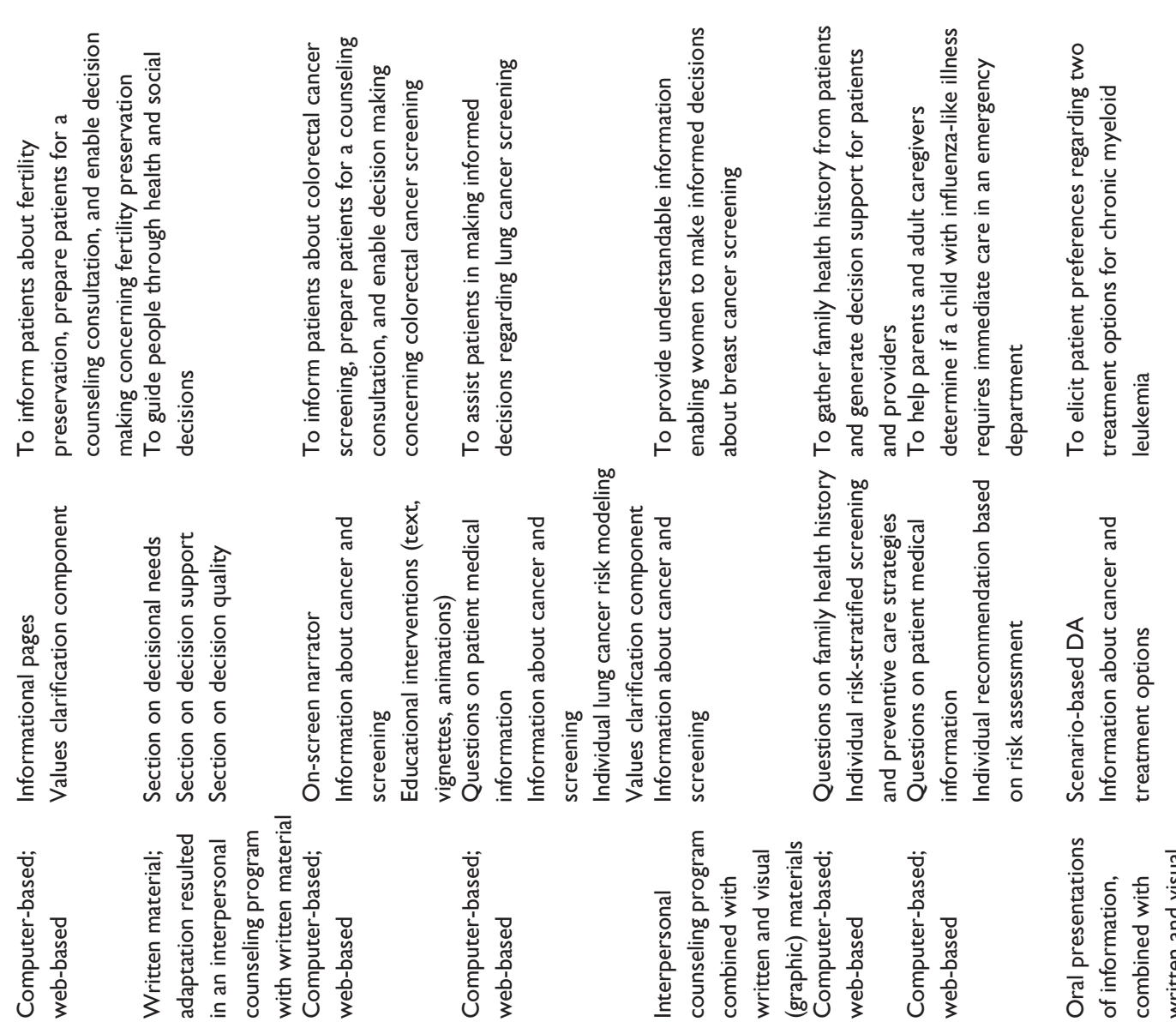

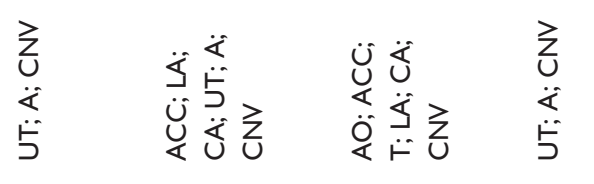

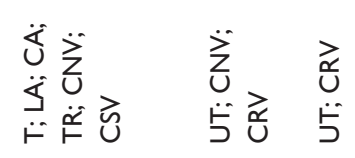

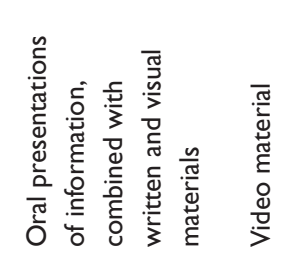

$\ddot{\leftrightarrow}$
$\ddot{\leftarrow}$
$\dot{5}$

$\times$

$\times$

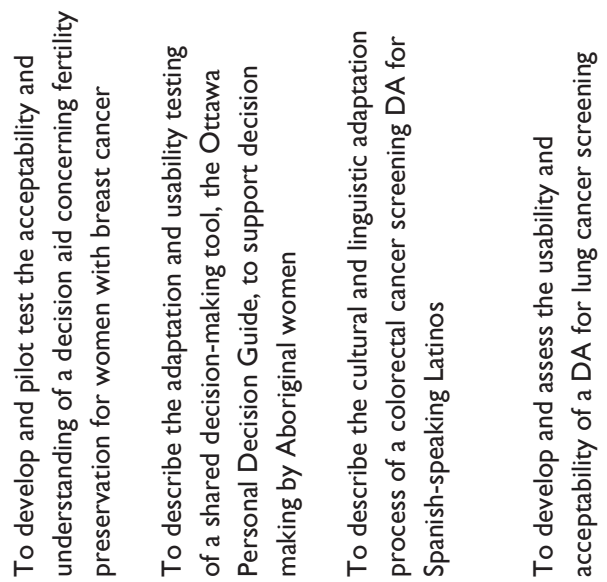

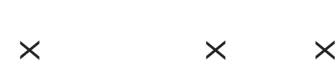

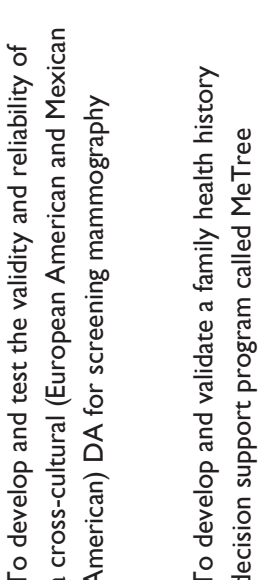

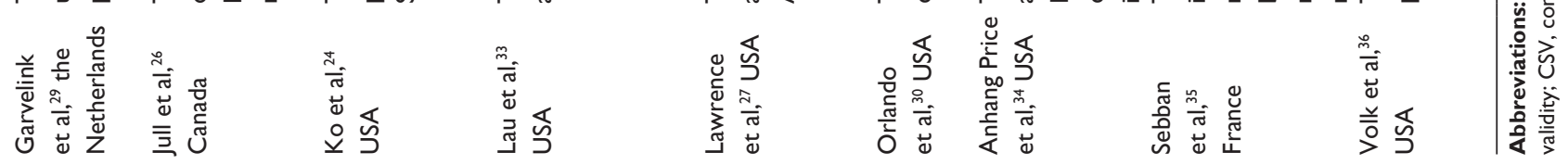

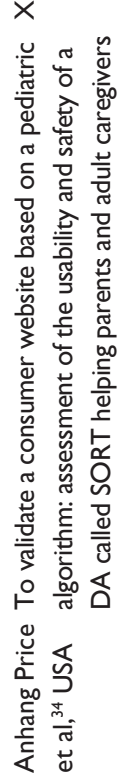

$\times$

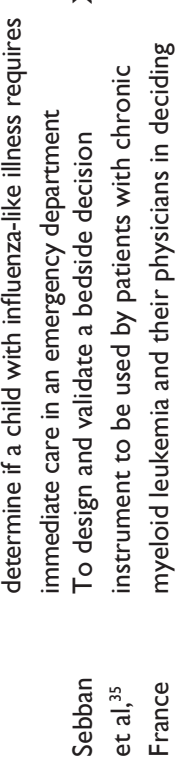

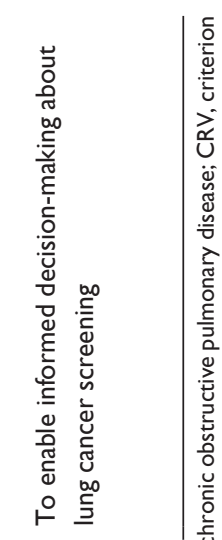

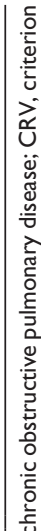


in a study involving an advisory group with representation from the cultural community. ${ }^{26}$ This preliminary step allowed the implementation of an adaptation process aligned with the community partners' sociocultural values. In addition, two focus groups allowed the authors to collect culturally relevant feedback about the original DA prior to its adaptation. Similarly, Ko et al asked community members for their feedback and input about how the content, format, graphics, and individuals portrayed in the DA could be adapted to increase relevance and message effectiveness. ${ }^{24}$

\section{Translation of the DA}

Four studies documented the procedure for translating a DA. ${ }^{24,25,27,28}$ Three used forward and back translation by experienced, bilingual translators working independently and with a translation committee..$^{25,27,28}$ The fourth study ${ }^{24}$ produced a direct translation of the initial prototype of the adapted DA during focus groups. The prototype was prepared by researchers and staff, who were native Spanish speakers, with a view to iteratively refine the translation throughout the adaptation process.

\section{Linguistic adaptation of the DA}

In parallel with or replacing the translation process (when the same language is spoken in both cultural contexts), the linguistic adaptation aims to ensure the equivalence of the words and expressions used between the original and adapted DA. In five studies, attention was paid to the appropriate literacy level of the adapted version versus that of the initial version. To operationalize this procedure, Berry et al asked the translators to use terminology applicable across nationalities of Spanish-speaking Latinos in the US. ${ }^{25}$ The use of linguistic nuances was also considered by Ko et al and Lawrence et al. ${ }^{24,27}$ Similarly, Coudeyre et al compared the equivalence of certain expressions (eg, translation of "meals on wheels" in French by "portage de repas", which could be back-translated into English by "meal porterage"). ${ }^{28}$ Jull et al also made some linguistic adaptations to reflect preferences and values in two communities - Aboriginal and Western speaking the same language. For instance, some statements in one section of the DA were rephrased using positive language instead of negative affirmations (eg, participants suggested "If you need to know more" instead of "If you feel you do NOT have enough facts"). ${ }^{26}$

\section{Cultural adaptation of the DA}

In addition to the attention paid to the wording of DAs, four studies described how the content of the DA was adapted to ensure a better fit with the new cultural context. Berry et al stressed that DA users should be able to identify or recognize themselves in the tool; hence, Spanish video clips were produced using bilingual Latino actors to play patients and physicians. ${ }^{25}$ Likewise, Ko et al adapted the content of the DA to take into consideration and integrate Latino sociocultural realities such as familism (the importance of family over individual interests), power distance and machismo. ${ }^{24}$ In another study, Coudeyre et al reported difficulty with adaptations related to the French health care system. ${ }^{28}$ For example, there is no equivalent for the concept of "district nurse" in France. Since "home care nurse" is the most frequent kind of ambulatory nurse (ie, a nurse who cares for patients in settings other than hospitals) in France, the expression "district nurse" was translated into French by "infirmière à domicile" (home care nurse), which represents a different reality in English. The format of a DA may also need adaptation. In a study by Jull et al, community members preferred to use the $\mathrm{DA}$ as a talking guide rather than a reflective tool because they found oral interaction with a decision coach to be a better fit with their own cultural approach to problem solving. ${ }^{26}$

\section{Usability testing}

Nine studies gathered information on how to improve DA structure, content, and format through user evaluation. Usability testing using a think aloud method - in which users are asked to share their impressions aloud while they are going through the DA - was reported by several authors as a way to identify potential issues in the format and content of the DA during individual interviews. ${ }^{25,26,29,30} \mathrm{~A}$ usability questionnaire addressing features of the DA can also be a way to identify usability issues. ${ }^{31}$ In all cases, this procedure was used by the authors to collect comments and identify issues with navigation, content comprehension and completeness, sociocultural appropriateness, proxy user problems; ${ }^{25}$ length of DA; ${ }^{29,32}$ amount of information; ${ }^{32}$ use of figures/ illustrations, ${ }^{29}$ comprehension of the content, design, layout, and messages conveyed; ${ }^{33}$ phrasing and clarity of the questions, usability of the interface (screen layout, skip patterns, fonts, button size, and other formatting features) ${ }^{30}$ and ease of understanding and answering the questions. ${ }^{34}$ Time of completion was also assessed in six studies. ${ }^{29-32,34,35}$

\section{DA acceptability}

DA acceptability was assessed in six studies. ${ }^{25,29,32,33,35,36}$ Aligned with the concept of information acceptability, ${ }^{37}$ the authors focused on the content presented in the DA (rather than on the format and structure of the DA). Garvelink et al 
documented qualitatively and quantitatively the difficulty in understanding the information and the relevance of the information presented in the DA during in-depth interviews. ${ }^{29}$ In two other studies, authors similarly documented the clarity and comprehensiveness of the written information in the $\mathrm{DA},{ }^{35}$ as well as graphic information such as charts. ${ }^{32}$ The authors of three studies quantified acceptability by means of a survey or questionnaire based on existing guidelines. ${ }^{25,33,36}$ Two studies ${ }^{26,35}$ also evaluated what could be described as ethical acceptability, ${ }^{38}$ referring to the value judgment made by potential users regarding the DA's desirability as an optimal solution for a specific context.

\section{Test-retest reliability}

Based on the operationalization of what constitutes a DA (ie, reflective tool rather than measurement tool), test-retest reliability was assessed in two studies evaluating the stability of the users' final decision, for repeated measures over time. ${ }^{27,35}$ Lawrence et al determined reliability (stability of the decision to get a mammography, measured in proportion) by administering the DA to 28 participants on two occasions, 1-2 weeks apart. ${ }^{27}$ Similarly, Sebban et al determined reliability (stability of the preferred treatment in chronic myeloid leukemia, measured by calculating an intraclass correlation coefficient) by administering the DA to 16 participants on two occasions scheduled 2 weeks apart. The two DAs were judged highly reliable. ${ }^{35}$

\section{Content validity}

Content validity was examined in eight studies as a way to evaluate the adequateness of the DA's focus and purpose. ${ }^{24-30,33}$ This procedure was used in five studies that asked for health specialists' feedback on the content. ${ }^{27-30,33}$ A simpler approach, namely through the assessment of face validity, was taken with potential or actual patients in seven of the studies..$^{24-27,29,30,33}$ To assess content validity (including face validity), a translation meeting was used by Coudeyre et al to obtain a common consensus on the final DA. ${ }^{28}$ In another study, feedback and suggestions concerning a DA prototype were collected from public health and health risk communication experts. ${ }^{33}$ Finally, a Delphi study with health care professionals, ${ }^{29}$ interviews with key stakeholders, ${ }^{25,30}$ focus groups, ${ }^{24,27}$ and review of the final DA with community representatives ${ }^{26}$ were some of the ways used to gather feedback later in the development process.

\section{Construct validity}

Two studies tested the degree to which users' final decision regarding a situation was consistent with specific hypotheses. ${ }^{27,35}$ In one case, probabilities presented in the DA to the participants were arbitrarily modified to verify if they changed their preferences in a predictable direction. ${ }^{27}$ In the other case, the understanding of the information provided, the quantity of information on the expressed choice, and the age of respondents were tested as three elements that might hypothetically affect final preferences. ${ }^{35}$

\section{Criterion validity}

Lastly, two studies evaluated the degree to which the recommendation of the DA was related to a "gold standard". 30,34 Orlando et al asked health specialists (genetic counselors) to review patients' pedigrees and compare them (ie, frequencies of matches and mismatches) to system recommendations (DA reports).$^{30}$ Based on their expertise, mismatches between these elements were documented. Anhang Price et al also assessed the adequacy of the recommendations compared to clinical "gold standards" (in this case, documented evidence in patient records); once again, DA recommendations were compared (exact 95\% CI) with patient records. ${ }^{34}$

\section{Core phases of the adaptation process}

Based on our analysis of the selected studies and the core procedures documented, we structured the procedures and suggest that the adaptation and validation process of patient DAs occurs in four iterative phases. These four phases aim to 1) explore the original DA and the new cultural context, 2) adapt the original DA to the new cultural context, 3) lab test the preliminary version of the adapted DA, and 4) field test the adapted DA in a real use context (see Figure 2).

This process starts with exploring the original DA and the new cultural context. Identifying the nature of the content of the DA and establishing relations between sections of the DA will inform what needs to be adapted to the new cultural context and how these adaptations may impact the DA. For instance, written, graphic, or video information sections may be modified according to the target population. This is followed by adapting the original DA to the new cultural context. The purpose of this phase is to consolidate findings from the previous phase and plan the procedures needed to produce an adapted version of the DA. Generally, translation, linguistic adaptation, and cultural adaptation are the procedures that transform the DA to fit the new cultural context. The inclusion of a steering committee (comprising representatives from the cultural community and professional translators, if necessary) in the early stages of the adaptation process could also inform the procedure to be undertaken. The adaptation and validation 


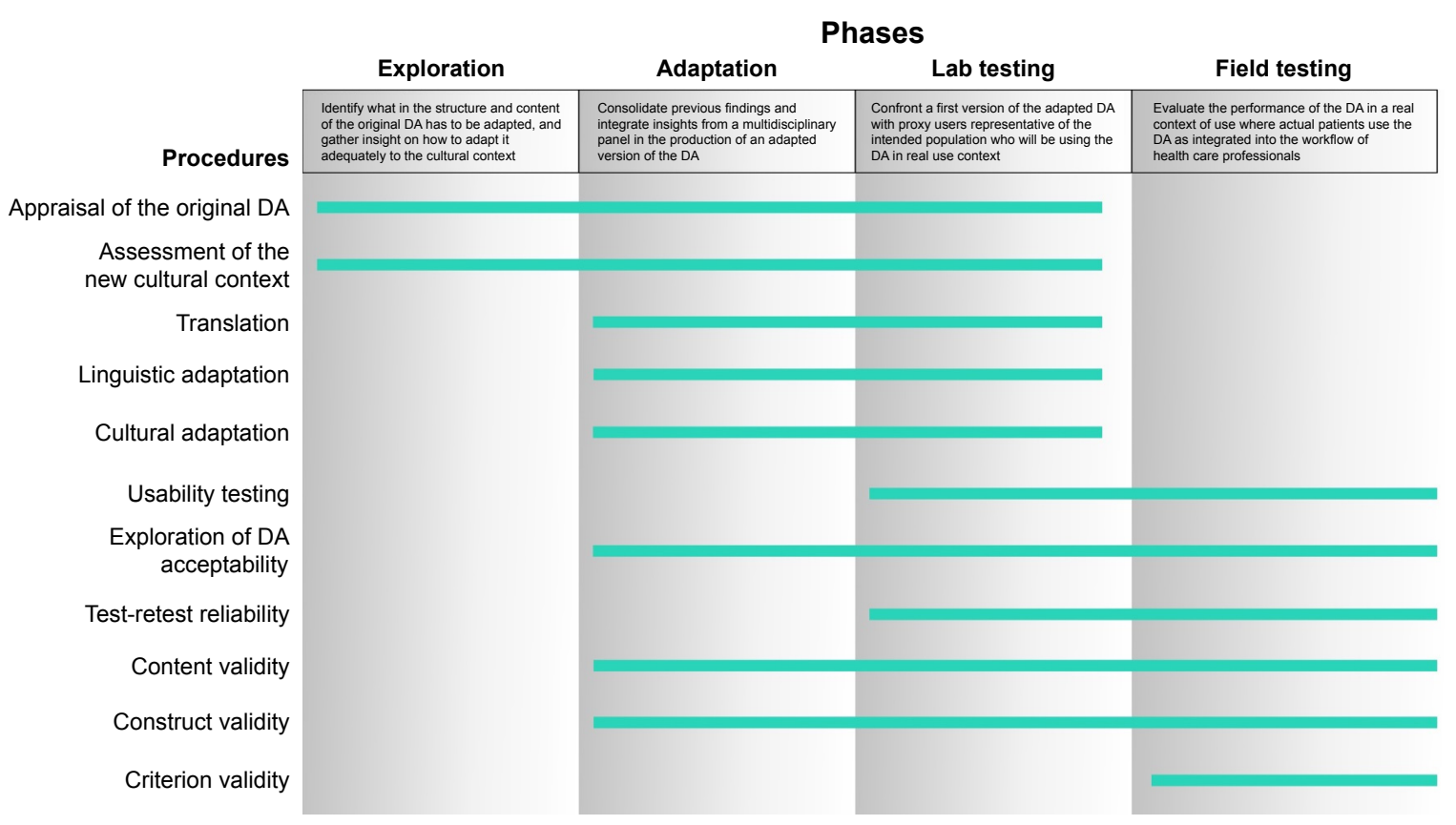

Figure 2 Flowchart of the four adaptation and validation phases and related procedures.

Abbreviation: DA, decision aid.

process is incomplete without testing a preliminary version of the adapted DA and testing the adapted DA in a real use context. The third phase is lab testing. The purpose of this phase is to present a preliminary version of the adapted DA to potential users, representative of the target population. This phase also involves gathering additional information to improve the fit between the DA and the cultural context. For instance, in addition to potential users, feedback on the preliminary version of the adapted DA can be collected via individual interviews with other stakeholders. Finally, prior to deploying the DA in a clinical context, the fourth phase is field testing. The purpose of this phase is to have real users evaluate the performance of the DA as it is integrated into practice or the workflow of health care professionals. Concerning field testing with potential users at the end of this phase, developers should have collected enough information to decide whether it is necessary to integrate new evidence and improve the adaptation or if they should consider the DA as adapted and valid in this cultural context.

All these phases are part of a continuum in which the adaptation and validation procedures overlap each other but are not restricted to a precise temporal order; instead of being linear, some phases may be repeated iteratively if necessary. Since the adaptation process could have impacted the content and structure of the DA, the process would be incomplete without the two testing phases, ensuring that appropriate decisions are made regarding implementation of the DA. This means that lab and field testing are mandatory, as is the case when a DA is developed..$^{10}$ The objective of adapting a DA is not to produce a similar version of the intervention but to create one that will likely achieve the same goal in another cultural context with another target population. Consequently, in addition to ensuring the DA is adapted adequately, this four-phase process opens the door to what has been called transcreation, defined as:

$[\ldots]$ the process of not only translating the text of written materials into another language, but also infusing culturally relevant context, photos, and themes (p. 92). ${ }^{39}$

Thus, instead of being rigid, this four-phase method is open to changes that can be iteratively incorporated, especially if content is added or the structure modified for cultural reasons.

\section{Discussion and implications}

This study is the first to address the cultural adaptation and validation process of patient DAs. The objective was to identify and document existing methods for cultural adaptation and validation of patient DAs.

In the selected studies, 11 key procedures were identified as steps to follow to adapt and validate DAs. However, the absence of guidelines for the adaptation and validation of patient DAs forced the authors of these studies to rely on the existing literature. Most of the literature addresses the 
selection and evaluation of health measurement instruments by means of standardized assessments ${ }^{14}$ and the cultural adaptation of related instruments by means of translation guidelines adapted to that specific type of health-related tool. ${ }^{12}$ Consequently, the adaptation and validation methods used in the studies reviewed might not have been really optimal or tailored for patient DAs. Most of the authors used psychometric language when developing or adapting questionnaires (ie, validity and reliability testing). Since the aim of patient DAs is to enhance decision making about health care options in a specific context for a target population, caution should be exercised in the ways these traditional methods are operationalized with DAs.

Since DAs are developed as reflective and orientation tools designed to facilitate the decision-making process around a health-related dilemma, patient DAs may be considered as interventions rather than measures. This is an important distinction that can impact how procedures are operationalized in the adaptation and validation process. For example, a test-retest procedure was conducted in two studies evaluating the stability of DA users' final decision for repeated measures over time..$^{27,35}$ One of the most common definitions of the test-retest procedure involves the concept of reliability and refers to the stability of the outcome for repeated measures. ${ }^{14}$ Because the user's opinion is not always the result of a measurement, we suggest referring to the term "preference stability" instead of test-retest reliability. Mokkink et al's definition could, however, be appropriate when users are invited to provide the DA with some information (eg, age, sex) and answer specific questions; the recommendations dispensed could then be compared for repeated measures. ${ }^{14}$

The validity tryptic known as construct, content, and criterion validity ${ }^{14}$ might also present some limitations when it comes to DAs. This classical definition of validity likely falls short when applied to DAs: What does "construct validity" mean when there is nothing per se to measure? What does "content validity" mean when the constructs to measure and the questions may vary depending on the context and the target population? And what does "criterion validity" mean when the outcome is a personal decision to be made by an individual? An exception is made with respect to DAs developed to provide tailored recommendations informed by experts' knowledge; the experts' recommendation representing the "gold standard" ${ }^{40}$ A more abstract perspective and a unified concept of validity defined in terms of meaning and values ${ }^{41}$ could potentially be applied to and help in the validation of patient DAs. Validity could:
[...] apply beyond interpretive and action inferences derived from test scores as ordinarily conceived, but also to inferences based on any means of observing or documenting consistent behaviors or attributes. ${ }^{41}$

This involves the appraisal of aspects of validity and results in a contextualized level of validity rather than an absolute binary validity. ${ }^{14}$

Since DAs can take numerous forms, ranging from paper leaflets to online decision support systems, a first consideration when adapting a DA is its format. The 11 procedures and four-phase process are discussed here without regard to the type of patient DA. However, each type of DA faces different issues when it comes to cultural adaptation. As part of the exploration phase, special attention must be paid to cultural adaptations that may be specific to each type of DA. For example, written material has to be adapted to fit with the target population's literacy level and idioms. Audio-video DAs have to be adapted to target the appropriate population so users can identify themselves in the materials and characters. For web-based DAs, the cultural adaptation process has to integrate elements related to the online interface as well as elements related to the programming of the website itself. Localization (ie, process through which a website is not only translated but also adapted to the cultural, technical, and administrative specificities of the country) or globalization (ie, strategy of constraints that must be adjusted in all local sites) are two approaches that could take cultural aspects into consideration in the development of online material. ${ }^{42}$

Finally, before starting to adapt an existing patient DA, other aspects to consider are the quality of the original DA and the need to conduct the whole adaptation process. The quality of the original DA must be assessed before undertaking this process by answering this first question: is this DA worth adapting? Guidelines for developing high-quality patient DAs (ie, IPDAS) can assist the selection process with a clear, detailed checklist of criteria to compare available DAs. With respect to the need to adapt, since this can be a time- and resource-consuming process, the decision to adapt should be based on theory about the new cultural context and ultimately on research evidence of the benefits of making an adapted DA available for decision making. The need to adapt can also be determined by answering this second question: is it worth adapting this DA? For instance, in contexts where language is not the main issue when using a DA (eg, new country with the same language), the extent to which cultural adaptation will make a difference in decision making should be explored. By involving experts and/or potential 
users, this exploration should aim at determining if a fit is possible without any adaptation in the new cultural context, if adaptations are appropriate and, if so, which ones, or if developing a totally new DA would be preferable.

\section{Limitations and strengths of the study}

Two main limitations should be noted. First, for practical reasons, the study was restricted to published articles pertaining to patient DAs. Consequently, we did not include studies in other areas, such as health education or clinical DAs, which could have been relevant to the implementation process of an integrated adaptation method. While findings from such studies could have provided additional insights, the content related to those areas was beyond the scope of our work. Second, the exclusion of studies published in languages other than English or French and the decision not to include gray literature (research material produced and made available outside the traditional academic publication and distribution channels) limited the review and could have ignored some pertinent sources of information (eg, reports, theses) that could have contributed to this work. The strengths of the study include the systematic nature of the review following clear guidelines for conducting scoping reviews, the clear inclusion criteria for selecting studies, and the use of rigorous methods. Verification was completed by two authors, and data synthetization was validated in discussions with all five authors.

\section{Conclusion}

Patient DAs are effective tools to foster patient engagement and support them when multiple options have to be weighed in order to make an informed health care choice. More and more DAs are being developed for different populations and various contexts of use; however, as with standardized measurement tools, many existing DAs could be adapted rather than developing new ones from the ground up. This study was undertaken because there are currently no guidelines to help researchers and clinicians when adapting patient DAs. This resulted in the identification of 11 main procedures for the adaptation and validation of DAs, and led to the suggestion of four core iterative phases structuring the adaptation process. Aligned with the scoping review methodology, future studies should focus on conducting a consultation exercise to inform and validate the four-phase method, procedures, and approaches described in this review. Prospective work will then consist of applying this method to the actual cultural adaptation process of a DA.

\section{Acknowledgments}

This work was supported by the AGE-WELL Network of Centres of Excellence (Grant \# AW CRP 2015-WP8.1). Drs Auger and Guay received salary support from the Fonds de Recherche en Santé du Québec (FRQS) and Dr Mortenson from the Canadian Institutes of Health Research (CIHR). This study also recognizes the financial support from the Centre for Interdisciplinary Research in Rehabilitation of Greater Montreal (CRIR) for the linguistic revision of this paper.

\section{Disclosure}

The authors report no conflicts of interest in this work.

\section{References}

1. Stacey D, Légaré F, Col NF, et al. Decision aids for people facing health treatment or screening decisions. Cochrane Database Syst Rev. 2014;(1):CD001431.

2. Roy B. Decision-aid and decision-making. Eur J Oper Res. 1990; 45(2-3):324-331.

3. O'Connor AM, Rostom A, Fiset V, et al. Decision aids for patients facing health treatment or screening decisions: systematic review. BMJ. 1999;319(7212):731-734.

4. Elwyn G, Frosch D, Thomson R, et al. Shared decision making: a model for clinical practice. J Gen Intern Med. 2012;27(10):1361-1367.

5. Coulter A, Ellins J. Effectiveness of strategies for informing, educating, and involving patients. BMJ. 2007;335(7609):24-27.

6. Elwyn G, Kreuwel I, Durand MA, et al. How to develop web-based decision support interventions for patients: a process map. Patient Educ Couns. 2011;82(2):260-265.

7. Tucker S, Brand C, O'Shea S, et al. An evaluation of the use of self assessment for the provision of community equipment and adaptations in English local authorities. Br J Occup Ther. 2011;74(3):119-128.

8. Alden DL, Friend J, Schapira M, Stiggelbout A. Cultural targeting and tailoring of shared decision making technology: a theoretical framework for improving the effectiveness of patient decision aids in culturally diverse groups. Soc Sci Med. 2014;105:1-8.

9. Estabrooks C, Goel V, Thiel E, Pinfold P, Sawka C, Williams I. Decision aids: are they worth it? A systematic review. J Health Serv Res Policy. 2001;6(3):170-182.

10. Coulter A, Stilwell D, Kryworuchko J, Mullen PD, Ng CJ, van der Weijden T. A systematic development process for patient decision aids. BMC Med Inform Decis Mak. 2013;13(Suppl 2):S2.

11. Vallerand RJ. Vers une méthodologie de validation trans-culturelle de questionnaires psychologiques: implications pour la recherche en langue française [Toward a methodology for the transcultural validation of psychological questionnaires: implications for research in the French language]. Can Psychol. 1989;30(4):662-680. French [with English abstract].

12. Beaton DE, Bombardier C, Guillemin F, Ferraz MB. Guidelines for the process of cross-cultural adaptation of self-report measures. Spine (Phila Pa 1976). 2000;25(24):3186-3191.

13. Guillemin F, Bombardier C, Beaton D. Cross-cultural adaptation of health-related quality of life measures: literature review and proposed guidelines. J Clin Epidemiol. 1993;46(12):1417-1432.

14. Mokkink LB, Terwee CB, Patrick DL, et al. COSMIN Checklist Manual. Amsterdam: VU University Medical Centre; 2009.

15. Wild D, Eremenco S, Mear I, et al. Multinational trials-recommendations on the translations required, approaches to using the same language in different countries, and the approaches to support pooling the data: the ISPOR Patient-Reported Outcomes Translation and Linguistic Validation Good Research Practices Task Force report. Value Health. 2009; 12(4):430-440. 
16. Kreuter MW, Lukwago SN, Bucholtz RD, Clark EM, SandersThompson V. Achieving cultural appropriateness in health promotion programs: targeted and tailored approaches. Health Educ Behav. 2003; 30(2):133-146.

17. Elwyn G, O'Connor AM, Bennett C, et al. Assessing the quality of decision support technologies using the International Patient Decision Aid Standards instrument (IPDASi). PLoS One. 2009;4(3):e4705.

18. Volk RJ, Llewellyn-Thomas H, Stacey D, Elwyn G. Ten years of the International Patient Decision Aid Standards Collaboration: evolution of the core dimensions for assessing the quality of patient decision aids. BMC Med Inform Decis Mak. 2013;13(Suppl 2):S1.

19. Arksey H, O’Malley L. Scoping studies: towards a methodological framework. Int J Soc Res Methodol. 2005;8(1):19-32.

20. Levac D, Colquhoun H, O'Brien KK. Scoping studies: advancing the methodology. Implement Sci. 2010;5:69.

21. Molenaar S, Sprangers MA, Postma-Schuit FC, et al. Interpretive review: feasibility and effects of decision aids. Med Decis Making. 2000;20(1):112-127.

22. Elo S, Kyngäs H. The qualitative content analysis process. J Adv Nurs. 2008;62(1):107-115.

23. Moher D, Liberati A, Tetzlaff J, Altman DG; PRISMA Group. Preferred reporting items for systematic reviews and meta-analyses: the PRISMA statement. Ann Intern Med. 2009;151(4):264-269.

24. Ko LK, Reuland D, Jolles M, Clay R, Pignone M. Cultural and linguistic adaptation of a multimedia colorectal cancer screening decision aid for Spanish-speaking Latinos. J Health Commun. 2014;19(2):192-209.

25. Berry DL, Halpenny B, Bosco JLF, Bruyere J Jr, Sanda MG. Usability evaluation and adaptation of the e-health Personal Patient ProfileProstate decision aid for Spanish-speaking Latino men. BMC Med Inform Decis Mak. 2015;15:56.

26. Jull J, Giles A, Minwaashin Lodge, The Aboriginal Women's Support Centre, Boyer Y, Stacey D. Cultural adaptation of a shared decision making tool with Aboriginal women: a qualitative study. BMC Med Inform Decis Mak. 2015;15:1.

27. Lawrence VA, Streiner D, Hazuda HP, Naylor R, Levine M, Gafni A A cross-cultural consumer-based decision aid for screening mammography. Prev Med. 2000;30(3):200-208.

28. Coudeyre E, Descamps S, Mc Intyre J, Boisgard S, Poiraudeau S, Lefevre-Colau MM. Traduction et adaptation culturelle française d'un outil d'aide à la décision pour orienter les patients opérés d'une arthroplastie totale de la hanche ou du genou [Translation and French cultural adaptation of a decision making tool for patients orientation after total hip or knee arthroplasty]. Ann Phys Rehabil Med. 2009;52(10):694-703. French [with English abstract].
29. Garvelink MM, ter Kuile MM, Fischer MJ, et al. Development of a decision aid about fertility preservation for women with breast cancer in the Netherlands. J Psychosom Obstet Gynaecol. 2013;34(4):170-178.

30. Orlando LA, Buchanan AH, Hahn SE, et al. Development and validation of a primary care-based family health history and decision support program (MeTree). N C Med J. 2013;74(4):287-296.

31. Akl EA, Grant BJ, Guyatt GH, Montori VM, Schünemann HJ. A decision aid for COPD patients considering inhaled steroid therapy: development and before and after pilot testing. BMC Med Inform Decis Mak. 2007;7:12.

32. Fatima S, Holbrook A, Schulman S, Park S, Troyan S, Curnew G. Development and validation of a decision aid for choosing among antithrombotic agents for atrial fibrillation. Thromb Res. 2016;145:143-148.

33. Lau YK, Caverly TJ, Cherng ST, et al. Development and validation of a personalized, web-based decision aid for lung cancer screening using mixed methods: a study protocol. JMIR Res Protoc. 2014;3(4):e78.

34. Anhang Price R, Fagbuyi D, Harris R, et al. Feasibility of web-based self-triage by parents of children with influenza-like illness: a cautionary tale. JAMA Pediatr. 2013;167(2):112-118.

35. Sebban C, Browman G, Gafni A, et al. Design and validation of a bedside decision instrument to elicit a patient's preference concerning allogenic bone marrow transplantation in chronic myeloid leukemia. Am J Hematol. 1995;48(4):221-227.

36. Volk RJ, Linder SK, Leal VB, et al. Feasibility of a patient decision aid about lung cancer screening with low-dose computed tomography. Prev Med. 2014;62:60-63.

37. O’Connor AM, Cranney A. User Manual-Acceptability. Ottawa, ON: Ottawa Hospital Research Institute; 2002.

38. Patenaude J, Legault GA, Beauvais J, et al. Framework for the analysis of nanotechnologies' impacts and ethical acceptability: basis of an interdisciplinary approach to assessing novel technologies. Sci Eng Ethics. 2015;21(2):293-315.

39. Simmons VN, Cruz LM, Brandon TH, Quinn GP. Translation and adaptation of smoking relapse-prevention materials for pregnant and postpartum Hispanic women. J Health Commun. 2010;16(1):90-107.

40. O'Keefe RM, Balci O, Smith EP. Validation of Expert System Performance. Department of Computer Science, Virginia Polytechnic Institute and State University; 1986.

41. Messick S. Validity of psychological assessment: validation of inferences from persons' responses and performances as scientific inquiry into score meaning. Am Psychol. 1995;50(9):741-749.

42. Tixier M. Globalization and localization of contents: evolution of major internet sites across sectors of industry. Thunderbird Int Bus Rev. $2005 ; 47(1): 15-48$

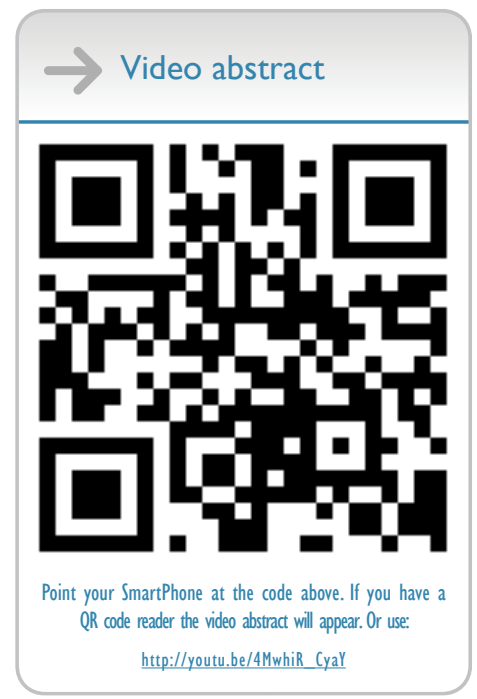


Patient Preference and Adherence

Dovepress

\section{Publish your work in this journal}

Patient Preference and Adherence is an international, peer-reviewed, open access journal that focuses on the growing importance of patient preference and adherence throughout the therapeutic continuum. Patient satisfaction, acceptability, quality of life, compliance, persistence and their role in developing new therapeutic modalities and compounds to optimize

clinical outcomes for existing disease states are major areas of interest for the journal. This journal has been accepted for indexing on PubMed Central. The manuscript management system is completely online and includes a very quick and fair peer-review system, which is all easy to use. Visit http://www. dovepress.com/testimonials.php to read real quotes from published authors.

Submit your manuscript here: http://www.dovepress.com/patient-preference-and-adherence-journal 